\title{
Preparation and Characterization of UV-Curable Cyclohexanone-Formaldehyde Resin and Its Cured Film Properties
}

\author{
Guang Yang, ${ }^{1}$ Hongqiang Li, ${ }^{1}$ Xuejun Lai, ${ }^{1}$ Yi Wang, ${ }^{1}$ Yifu Zhang, ${ }^{2}$ and Xingrong Zeng ${ }^{1}$ \\ ${ }^{1}$ College of Materials Science and Engineering, South China University of Technology, Guangzhou 510640, China \\ ${ }^{2}$ Yueyang Intech Synthetic Material Co., Ltd., Yueyang 414009, China \\ Correspondence should be addressed to Hongqiang Li; hqli1979@gmail.com and Xingrong Zeng; psxrzeng@gmail.com
}

Received 11 July 2014; Revised 29 September 2014; Accepted 22 October 2014; Published 11 November 2014

Academic Editor: Jose Ramon Leiza

Copyright (C) 2014 Guang Yang et al. This is an open access article distributed under the Creative Commons Attribution License, which permits unrestricted use, distribution, and reproduction in any medium, provided the original work is properly cited.

UV-curable cyclohexanone-formaldehyde (UVCF) resin was prepared with cyclohexanone-formaldehyde (CF) resin, isophorone diisocyanate (IPDI), and pentaerythritol triacrylate (PETA) as base substance, bridging agent, and functional monomer, respectively. The structure of UVCF was characterized by Fourier transform infrared spectroscopy (FT-IR), ${ }^{1} \mathrm{H}$-nuclear magnetic resonance spectroscopy $\left({ }^{1} \mathrm{H}-\mathrm{NMR}\right)$, and gel permeation chromatography (GPC). The viscosity and photopolymerization behavior of the UV-curable formulations were studied. The thermal stability and mechanical properties of the cured films were also investigated. The results showed that UVCF resin was successfully prepared, the number of average molecular weight was about 2010, and its molecular weight distribution index was 2.8. With the increase of UVCF resin content, the viscosity of the UVcurable formulations increased. After exposure to UV irradiation for $230 \mathrm{~s}$, the photopolymerization conversion of the UV-curable formulations was above $80 \%$. Moreover, when the UVCF content was $60 \%$, the formulations had high photopolymerization rate, and the cured UVCF films showed good thermal stability and mechanical properties.

\section{Introduction}

Ketone-aldehyde (KA) resin, an important resins synthesized with ketone and aldehyde, is well known as multifunctional additives in coatings and inks $[1,2]$. Owing to the existence of carbonyl and hydroxyl groups, in the molecular structure, KA resin has good compatibility with coating or ink resins, and can be used as dispersant for pigments in the coatings and inks [3-5]. Meanwhile, KA resin can endow the products with high hardness and good adhesion to various substrates. In addition, the saturated chain in KA resin made contributions to the high gloss of the coating film $[6,7]$. Therefore, the preparation of $\mathrm{KA}$ resin and its application in coatings and inks have been paid more and more attentions [8-10]. Zhang et al. synthesized the urea-isobutyraldehyde-formaldehyde (UIF) resins by the condensation of urea, isobutyraldehyde, and formaldehyde. The results showed that the UIF resins had good yellowing resistance, UV resistance, and solubility in common organic solvents [11]. A new melamine-formaldehyde-butanone (MFB) resin was prepared by Glowacz-Czerwonka and Kucharski [12], and they found that the coatings with MFB resin had transparent appearance, high hardness, and good resistance against boiling water.

Nowadays, due to its advantages of fast curing rate, energy saving, and environment protection, UV-curing has been considered to be one of the most promising technologies [1315]. For example, compared to traditional thermal-curable coatings, UV-curable coatings always show better comprehensive performance such as excellent film-forming property and high thermal stability. However, most of the KA resins have no $\mathrm{C}=\mathrm{C}$ double bonds to react with $\mathrm{UV}$-curable resin and it is easily to migrate from the UV-curable coatings, which will lead to the decrease of the mechanical properties and solvent-resistant properties [16]. Therefore, it is necessary to introduce the curable groups into the structure of KA resin for UV-curing. Mishra et al. [17] prepared a radiationcurable resin through modifying the carbonyl-hydrogenated 
KA resin with isophorone diisocyanate (IPDI) and hydroxy acrylate, and the UV-cured films showed good resistance to organic solvents. Thus, the carbonyl and hydroxyl reactive groups in $\mathrm{KA}$ resin provide potential modification with curable groups, which allows the KA resin to be cured under radiation condition.

In this study, a novel UV-curable cyclohexanoneformaldehyde (UVCF) resin was synthesized with cyclohexanone-formaldehyde (CF) resin, isophorone diisocyanate (IPDI), and pentaerythritol triacrylate (PETA) as base substance, bridging agent, and functional monomer, respectively. The UV-curable formulations were composed of UVCF resin, tripropylene glycol diacrylate (TPGDA), and 2-hydroxy2-methyl-phenyl-propan-1-one (Darocur 1173). The UVCF resin was characterized by gel permeation chromatography (GPC), Fourier transform infrared spectroscopy (FT-IR), and ${ }^{1} \mathrm{H}$-nuclear magnetic resonance spectroscopy $\left({ }^{1} \mathrm{H}\right.$-NMR). The effects of UVCF content on the viscosity and photopolymerization behavior of the UV-curable formulations were studied. The thermal stability and mechanical properties of the cured films were also investigated.

\section{Experimental}

2.1. Materials. Cyclohexanone-formaldehyde (CF) resin was acquired from Intech Innovative Materials Company (China). Isophorone diisocyanate (IPDI) was supplied by Degussa Company (Germany). Pentaerythritol triacrylate (PETA) and tripropylene glycol diacrylate (TPGDA) were received from AGI Corporation Company (Taiwan). Butyl acetate was provided by Guanglianjin Chemical Industry Company (China). Dibutyltin dilaurate (DBTDL) was obtained from Shanghai Lingfeng Chemical Reagent Company (China). Hydroquinone was purchased from Tianjin Kemiou Chemical Reagent Company (China). 2-Hydroxy-2-methyl-phenylpropan-1-one (Darocur 1173) was supplied by Ciba Geigy Company (Switzerland). Hexane and acetone were purchased from Guangdong Guanghua Chemical Factory (China).

2.2. Synthesis of UVCF Resin. UVCF resin was synthesized by two steps as shown in Figure 1. Firstly, CF resin $(50 \mathrm{~g})$ and butyl acetate $(143 \mathrm{~g})$ were added into a four-neck glass reactor equipped with a reflux condenser, blender, and thermometer under dry nitrogen gas. When $\mathrm{CF}$ resin was dissolved, DBTDL $(0.54 \mathrm{~g})$ and IPDI $(22.2 \mathrm{~g}, 0.1 \mathrm{~mol})$ were added dropwise into the reactor and the temperature was heated to $50^{\circ} \mathrm{C}$. The NCO value during the reaction was determined using the dibutylamine back-titration method every half an hour. When the NCO\% decreased to about 50\%, PETA ( $35.8 \mathrm{~g}$, $0.12 \mathrm{~mol}$ ) and hydroquinone $(0.5 \mathrm{wt} \%)$ were dropwise into the reactor and the temperature raised to $60^{\circ} \mathrm{C}$. The reaction continued until $\mathrm{NCO} \%$ was below $0.5 \%$. The reactant was washed with distilled water and then dissolved in acetone and purified via precipitation method by hexane. Finally, after drying, under a vacuum drying oven at $40^{\circ} \mathrm{C}$, the light yellow powder product was obtained.
TABLE 1: Formulations of UV-cured films.

\begin{tabular}{lccc}
\hline Samples & UVCF (wt\%) & $\begin{array}{c}\text { TPGDA } \\
\text { (wt\%) }\end{array}$ & $\begin{array}{c}\text { Darocur 1173 } \\
\text { (wt\%) }\end{array}$ \\
\hline $\mathrm{S}_{1}$ & 20 & 75 & 5 \\
$\mathrm{~S}_{2}$ & 40 & 55 & 5 \\
$\mathrm{~S}_{3}$ & 60 & 35 & 5 \\
$\mathrm{~S}_{4}$ & 80 & 15 & 5 \\
$\mathrm{~S}_{5}$ & 95 & 0 & 5 \\
\hline
\end{tabular}

2.3. Preparation of UV-Cured Films. UVCF resin and Darocur 1173 were dissolved in TPGDA under magnetic stirring for about $15 \mathrm{~min}$, and the UV-curable formulation was obtained. Subsequently, the formulation was coated on tinplate by an applicator with a $20 \mu \mathrm{m}$ gap and cured for $2 \mathrm{~min}$ under UV radiation $(365 \mathrm{~nm}, 2 \mathrm{~kW}, 80 \mathrm{~W} / \mathrm{cm}$; Shenzhen Nengjia Automation Equipment Co., China). The detailed formulations of UV-cured films were given in Table 1 .

\subsection{Characterization}

2.4.1. Size Exclusion Chromatography (SEC). The molecular weight and molecular weight distribution of samples were determined with an equipment with a Waters 1515 pump and Waters 2414 differential refractive index detector (Waters, USA) using two columns HR4E and HR5 at $35^{\circ} \mathrm{C}$, and tetrahydrofuran was used as the mobile phase at an elution rate of $1.0 \mathrm{~mL} / \mathrm{min}$. The calibration was made with low polydispersity polystyrene samples.

2.4.2. Fourier Transform Infrared (FT-IR) Spectroscopy. FT-IR spectra were obtained from a Bruker Tensor 27 spectrometer (Bruker, Germany) in the range of $4000-400 \mathrm{~cm}^{-1}$ by $\mathrm{KBr}$ pellets method.

2.4.3. ${ }^{1} \mathrm{H}$-Nuclear Magnetic Resonance Spectroscopy $\left({ }^{1} \mathrm{H}\right.$ NMR). ${ }^{1} \mathrm{H}-\mathrm{NMR}$ spectrum was recorded on a DRX400 NMR Spectrometer (Bruker, Germany) at $25^{\circ} \mathrm{C}$ using $\mathrm{CDCl}_{3}$ as solvent and TMS as internal standard substance.

2.4.4. Viscosity. The viscosity was measured by an NDJ-7 model spinning viscometer (Shanghai Precision \& Scientific Instrument Company, China), with a speed of $750 \mathrm{rpm} / \mathrm{min}$ at different temperature.

2.4.5. Conversion of $C=C$ Bonds. The percent conversion of $\mathrm{C}=\mathrm{C}$ bonds in the curing process was calculated by the area of the characteristic absorption peak of UV formulations via FT-IR according to the method in the literature, and the conversion $(X)$ of $\mathrm{C}=\mathrm{C}$ bonds was calculated according to the following equation [18]:

$$
X(\%)=\frac{\left(A_{810} / A_{1724}\right)_{0}-\left(A_{810} / A_{1724}\right)_{t}}{\left(A_{810} / A_{1724}\right)_{0}} \times 100
$$



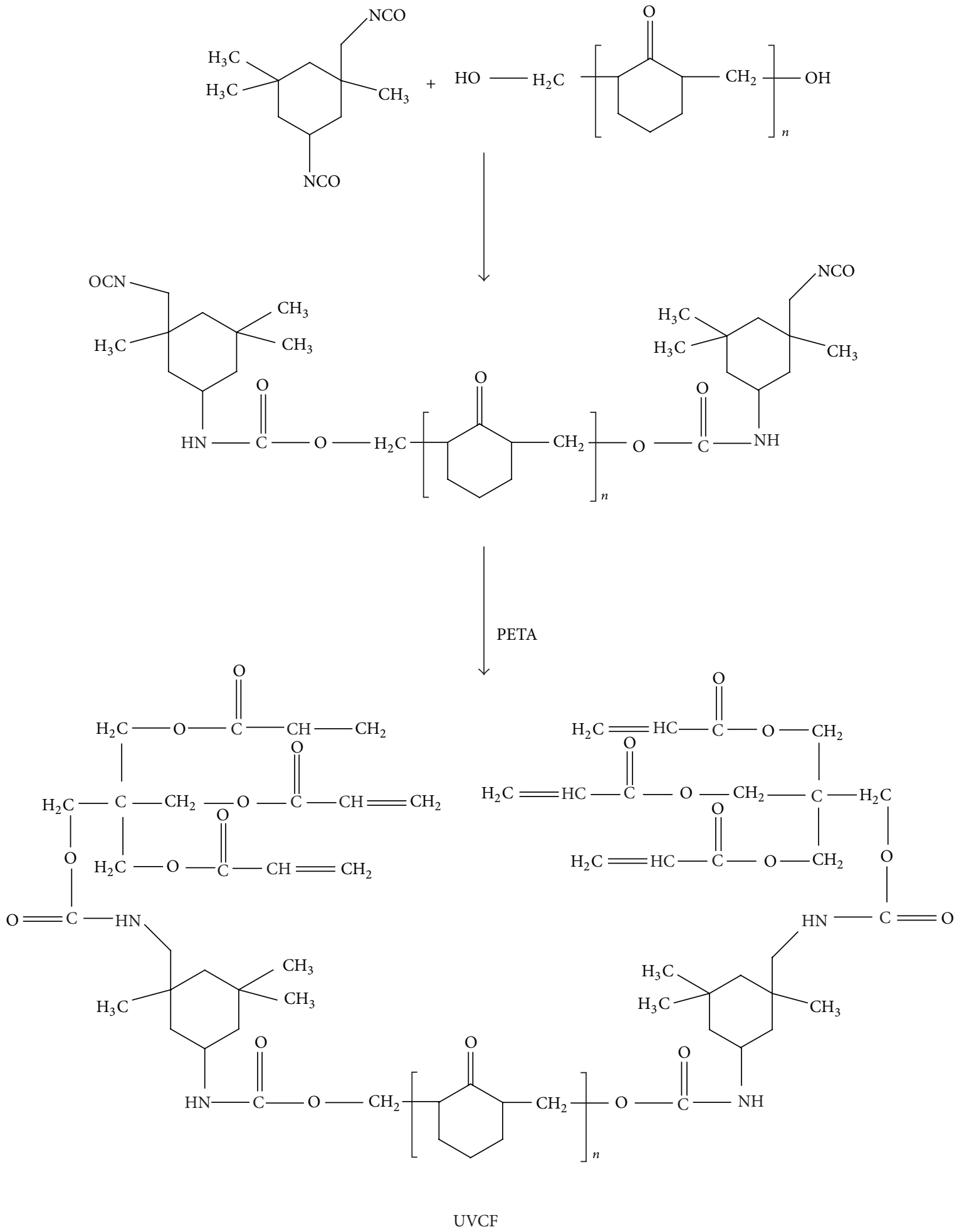

FIGURE 1: Schematic illustration for the synthesis of UVCF. 


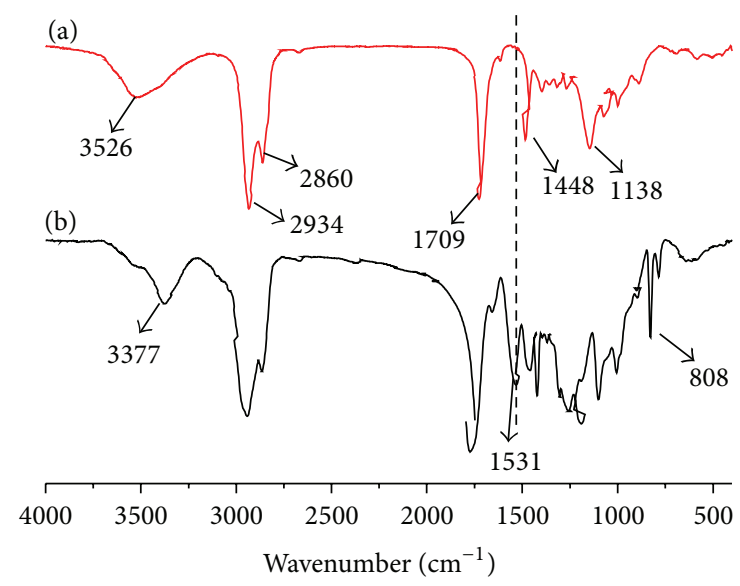

FIGURE 2: FT-IR spectra of CF resin (a) and UVCF resin (b).

where $A_{810}$ and $A_{1724}$ were the calibration peak area at 810 and $1724 \mathrm{~cm}^{-1}$ attributed to in-plane bending vibration of $\mathrm{C}-$ $\mathrm{H}$ in $\mathrm{C}=\mathrm{C}$ double bonds and stretching vibration of $\mathrm{C}=\mathrm{O}$ in urethane unit, respectively. $\left(A_{810} / A_{1724}\right)_{0}$ is the ratio of $A_{810}$ to $A_{1724}$ before UV radiation, and $\left(A_{810} / A_{1724}\right)_{t}$ is the ratio of $A_{810}$ to $A_{1724}$ at different UV exposure time.

2.4.6. Thermogravimetry (TG). TG measurements were obtained from a thermal gravimetric analysis (NETZSCH STA 449C, Germany) under nitrogen atmosphere at a heating rate of $5^{\circ} \mathrm{C} \cdot \mathrm{min}^{-1}$ from $30^{\circ} \mathrm{C}$ to $600^{\circ} \mathrm{C}$.

2.4.7. Mechanical Properties. The mechanical properties such as adhesive force, flexibility, pendulum hardness, and impact strength were measured according to the method of GB/T 9286-1998, GB/T 1731-1993, GB/T 1730-1993, and GB/T 17321993, respectively.

\section{Results and Discussion}

3.1. FT-IR Analysis. FT-IR spectra of CF resin and UVCF resin are shown in Figure 2. In the spectrum of CF resin, the bands at $3526 \mathrm{~cm}^{-1}$ were the stretching vibration absorption peaks of $-\mathrm{OH}$ groups on $\mathrm{CF}$ resin. The peaks at $2934 \mathrm{~cm}^{-1}$ and $2860 \mathrm{~cm}^{-1}$ were attributed to the asymmetric stretching vibration and symmetrical stretching vibration of $-\mathrm{CH}_{2}-$, and the peak at $1448 \mathrm{~cm}^{-1}$ was attributed to the deformation vibration of $-\mathrm{CH}_{2}-$. The bands located at $1709 \mathrm{~cm}^{-1}$ and $1138 \mathrm{~cm}^{-1}$ were attributed to the stretching vibration of $\mathrm{C}=\mathrm{O}$ and symmetrical stretching vibration of $\mathrm{C}-\mathrm{O}$, respectively. Compared with the FT-IR spectrum of CF resin, in Figure 2(b), new peaks of $-\mathrm{NH}$ group at $3377 \mathrm{~cm}^{-1}$ and $1531 \mathrm{~cm}^{-1}$ appeared, and the intensity of the peak attributed to - $\mathrm{OH}$ groups decreased obviously. Moreover, no absorption at $2270 \mathrm{~cm}^{-1}$ for -NCO group could be determined, indicating that IPDI had been reacted completely [19]. In addition, the weak peaks at $3100-3000 \mathrm{~cm}^{-1}$ and strong peak at $810 \mathrm{~cm}^{-1}$ attributed to the stretching vibration and in-plane bending vibration of $\mathrm{C}-\mathrm{H}$ in $\mathrm{C}=\mathrm{C}$ double bonds were observed in the
TABLE 2: Molecular weight and molecular weight distribution of CF resin and UVCF resin.

\begin{tabular}{lccc}
\hline Samples & $M_{n}(\mathrm{~g} / \mathrm{mol})$ & $M_{w}(\mathrm{~g} / \mathrm{mol})$ & PDI \\
\hline CF & 650 & 820 & 1.2 \\
UVCF & 2010 & 5628 & 2.8 \\
\hline
\end{tabular}

spectrum of UVCF, which conformed that PETA had been grafted onto CF resin.

3.2. ${ }^{1} \mathrm{H}$-NMR Analysis. Figure 3 showed ${ }^{1} \mathrm{H}-\mathrm{NMR}$ spectrum of UVCF resin. As shown in Figure 3, the peaks at 1.4$2.0 \mathrm{ppm}$ and $0.9-1.0 \mathrm{ppm}$ were attributed to the protons on methylene and methyl groups, respectively [20,21]. The peaks located at $4.0-4.2 \mathrm{ppm}$ were attributed to $\mathrm{H}$ atoms of $-\mathrm{O}-$ $\mathrm{CH}_{2}-$ groups. In addition, the peaks in the range of 5.8$6.5 \mathrm{ppm}$ were attributed to the protons from $\mathrm{C}=\mathrm{C}$ double bonds in PETA $[22,23]$. Because the obtained UVCF had been purified, it can be further concluded that PETA had been grafted onto $\mathrm{CF}$ resin, and the results of ${ }^{1} \mathrm{H}-\mathrm{NMR}$ were in accord with those of FT-IR.

3.3. SEC Analysis. The molecular weight and molecular weight distributions of CF resin and UVCF resin were determined by SEC and the results are shown in Figure 4 and Table 2. It can be observed that the number average molecular weight $\left(M_{n}\right)$ of CF resin was 650, and its molecular weight distributions (PDI) were 1.2. After being modified with IPDI and PETA, the $M_{n}$ of UVCF resin increased to 2010, which was close to the theory value. The PDI increased to 2.8 from 1.2 , indicating that there was unreacted PETA in the system.

3.4. Viscosity. The viscosity of the UVCF formulations had big effect on the liquid mobility, film-forming property, photopolymerization behavior, and the final mechanical properties of the cured film. Therefore, the viscosity should be adjusted to appropriate value before UV-curing. Figure 5 shows the viscosity of the formulations at different temperatures. In Figure 5, when the temperature was $30^{\circ} \mathrm{C}$, the viscosity of UVCF formulation with $20 \%$ content of UVCF was about $1500 \mathrm{mPa} \cdot \mathrm{s}$. With the increase of UVCF content, the viscosity increased obviously. When the UVCF content reached $80 \%$, the viscosity increased to $18000 \mathrm{mPa} \cdot \mathrm{s}$, which was mainly owing to the high inter-/intramolecular force of -NHCOO- groups and -COO-groups in UVCF. However, when the temperature increased from $30^{\circ} \mathrm{C}$ to $75^{\circ} \mathrm{C}$, the viscosity of all formulations decreased to a low value of $200-$ $300 \mathrm{mPa} \cdot \mathrm{s}$, which might be due to the decrease of inter-/ intramolecular force and the increase of molecular motion [24].

3.5. Photopolymerization Behavior. Real time FT-IR was used to study the photopolymerization behavior of UVCF formulations under different UV irradiation time. As shown in Figure 6, before UV irradiation, the absorbance peak at about $810 \mathrm{~cm}^{-1}$ that attributed to $\mathrm{C}-\mathrm{H}$ vibration from $\mathrm{C}=\mathrm{C}$ double bonds was strong. With the increase of UV irradiation time, 


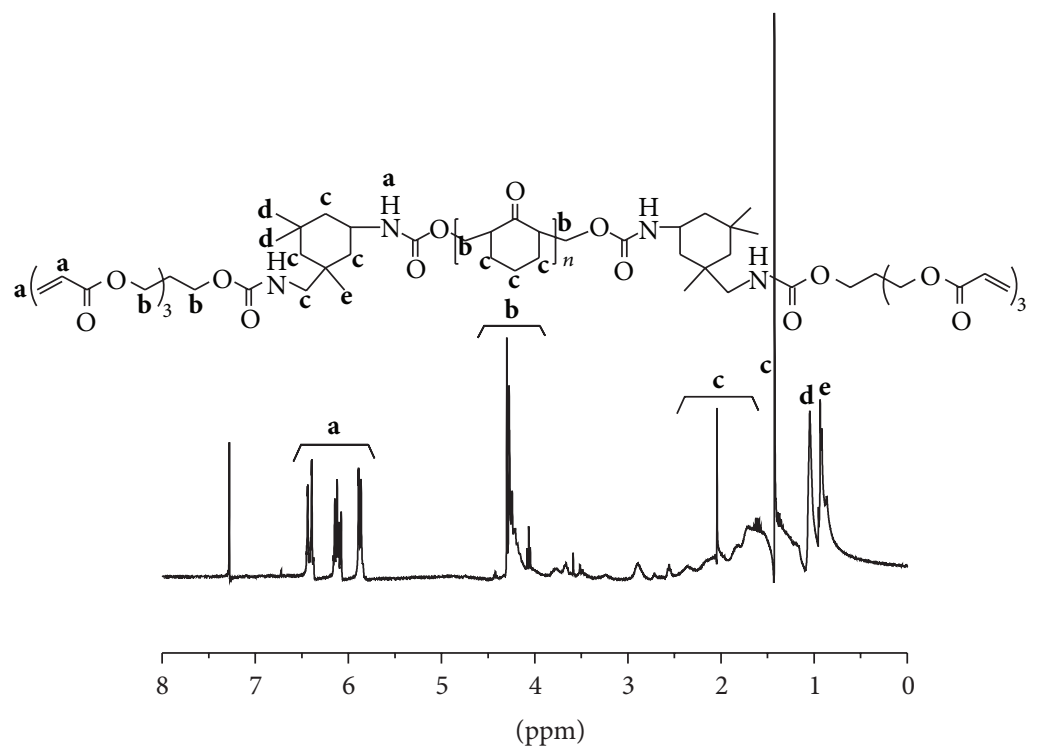

FIgURE $3:{ }^{1} \mathrm{H}-\mathrm{NMR}$ spectrum of UVCF resin.

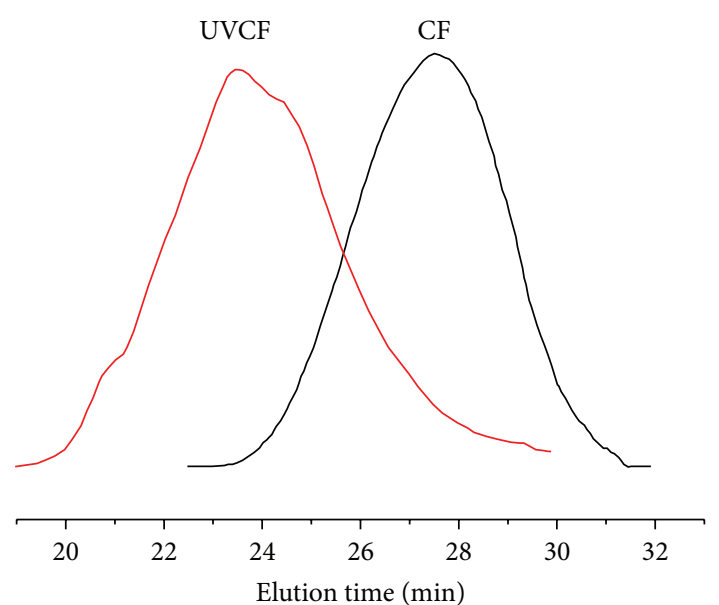

FIGURE 4: SEC curves of CF resin and UVCF resin. the $\mathrm{C}=\mathrm{C}$ double bonds in the UVCF began to react and the absorbance peak at $810 \mathrm{~cm}^{-1}$ became weak. When the irradiation time was $230 \mathrm{~s}$, the peaks at $810 \mathrm{~cm}^{-1}$ almost disappeared, indicating that the polymerization had been completely finished [18]. Therefore, the appropriate irradiation time was about $230 \mathrm{~s}$.

3.6. Effect of UVCF Content on Photopolymerization Conversion. Figure 7 shows the effect of UV irradiation time on the conversion of UV-curable formulations. As shown in Figure 7, with the increase of irradiation time, the conversions increased rapidly, and when the irradiation time was about 230 s, except the UV-curable formulation with $95 \%$ content of UVCF, the conversions of the other formulations all exceed $80 \%$ and reached to the maximum value. However, with the further increase of irradiation time, the conversion

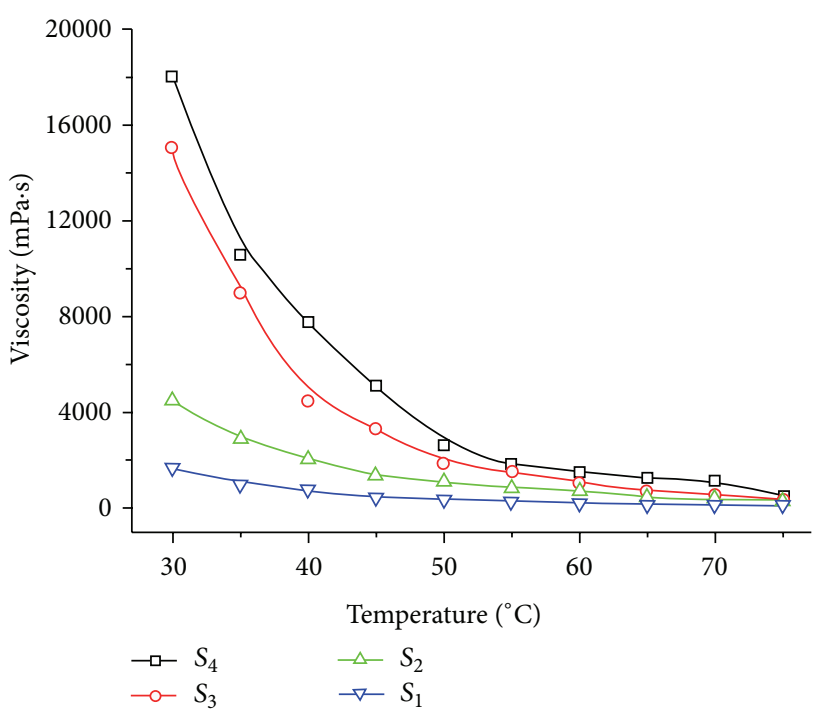

FIGURE 5: Viscosity of UVCF formulations at different temperatures.

increased a little. These results were consistent with the analysis of the real time FT-IR. In fact, in the later stage of the polymerization, some of the $\mathrm{C}=\mathrm{C}$ double bonds might be trapped by the high cross-linking structures [25], and the active free radical from the UVCF was restricted to react completely, so the conversion under UV-curing condition was difficult to reach $100 \%$. In addition, as shown in Figure 7, compared with other formulations, the UVCF formulation with $60 \%$ content of UVCF had higher photopolymerization rate and final conversion. The reasons could be explained as follows: when the UVCF content was low, the collision odds between reactive chains were little and it needed enough time to photopolymerized completely. When UVCF content was high, the viscosity of the formulations drastically increased, which led to 


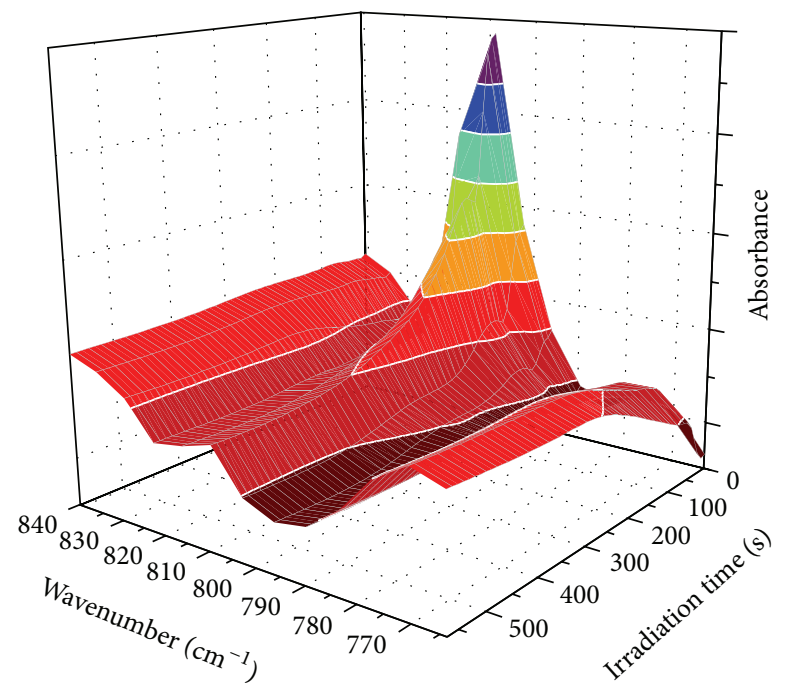

FIGURE 6: 3D profile of UVCF formulations at bands of $810 \mathrm{~cm}^{-1}$ was recorded under different UV irradiation time by real time FT-IR.

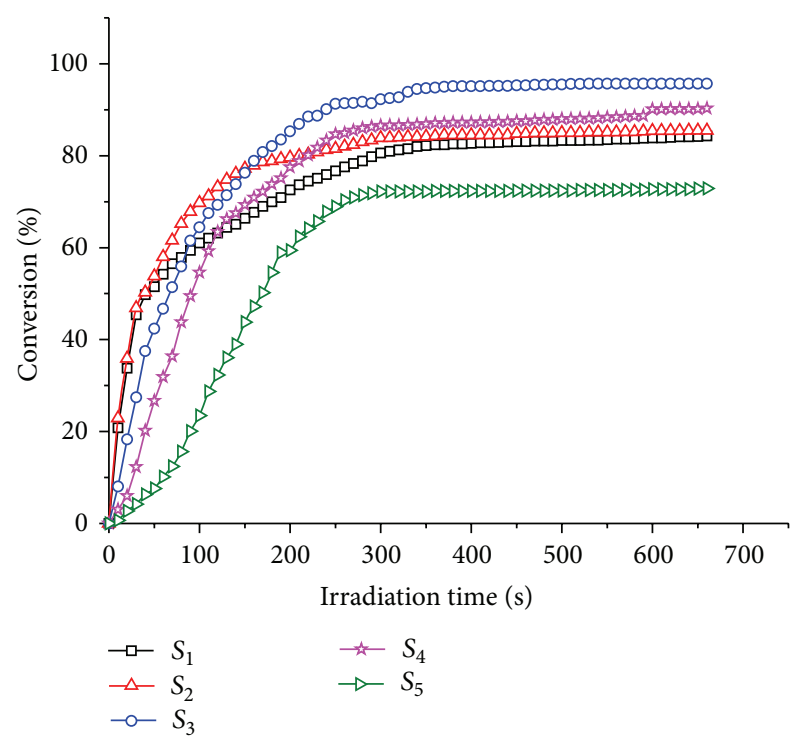

FIGURE 7: Effect of UV irradiation time on the conversion of UVcurable formulations with different UVCF content.

the limited mobility of reactive chains. Moreover, with high double bonds concentration, the photopolymerization easily occurred on the surface and formed the cross-linking structures, which might affect the mobility of reactive chain and the entrance of UV irradiation into the cured film [26].

3.7. Thermal Stability. TG curves of CF, UVCF, and the cured film of the formulation $S_{3}$ with $60 \%$ content of UVCF are shown in Figure 8. The initial decomposition temperature $\left(T_{i}\right)$ was defined as the temperature with $5 \%$ mass loss. From TG curves of CF and UVCF, the $T_{i}$ was at about $222^{\circ} \mathrm{C}$ and $169^{\circ} \mathrm{C}$, respectively. The continuous mass loss of UVCF before $250^{\circ} \mathrm{C}$ might be caused by the break of the $\mathrm{C}-\mathrm{O}$ bonds from the urethane groups [27]. The temperatures of maximum

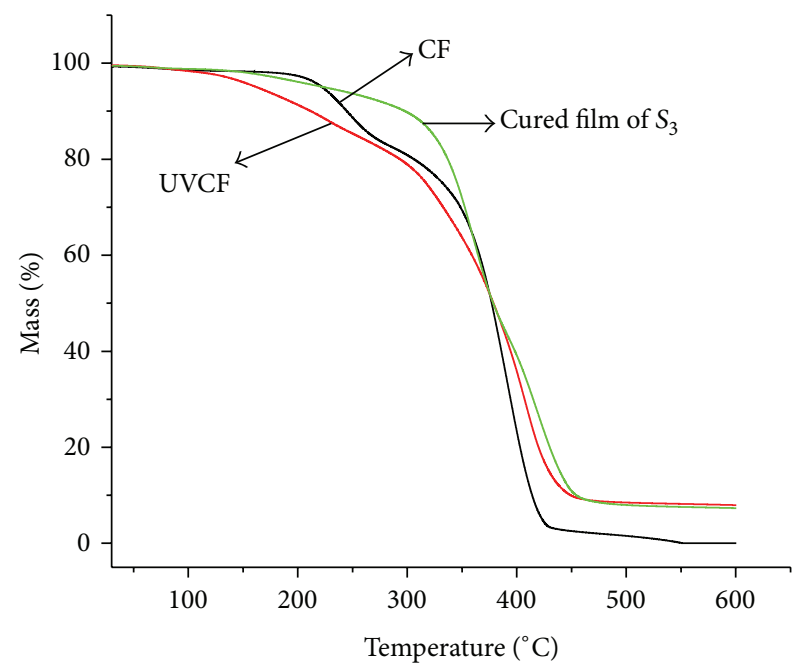

FIgURE 8: TG curves of CF, UVCF, and cured film of $S_{3}$.

TABLE 3: Mechanical properties of cured UVCF films.

\begin{tabular}{lcccc}
\hline Samples & $\begin{array}{c}\text { Pendulum } \\
\text { hardness } \\
\text { (time ratio) }\end{array}$ & $\begin{array}{c}\text { Flexibility } \\
(\mathrm{mm})\end{array}$ & $\begin{array}{c}\text { Adhesive } \\
\text { force (grade) }\end{array}$ & $\begin{array}{c}\text { Impact } \\
\text { strength } \\
(\mathrm{kg} \cdot \mathrm{cm})\end{array}$ \\
\hline $\mathrm{S}_{1}$ & 0.26 & 3 & 1 & 35 \\
$\mathrm{~S}_{2}$ & 0.34 & 4 & 0 & 38 \\
$\mathrm{~S}_{3}$ & 0.61 & 4 & 1 & 42 \\
$\mathrm{~S}_{4}$ & 0.48 & 5 & 1 & 34 \\
$\mathrm{~S}_{5}$ & 0.52 & 10 & 1 & 31 \\
\hline
\end{tabular}

weight loss rate $\left(T_{\max }\right)$ of CF and UVCF were at $300-320^{\circ} \mathrm{C}$. For cured UVCF formulation, with $60 \%$ content of UVCF, $T_{i}$ and $T_{\max }$ were at $250^{\circ} \mathrm{C}$ and $380^{\circ} \mathrm{C}$, respectively. Compared with CF and UVCF, the obvious increase of $T_{\max }$ of the cured UVCF formulation was mainly due to the formation of crosslinking structure.

3.8. Mechanical Properties. The mechanical properties of cured UVCF films with different UVCF content are listed in Table 3. As shown in Table 3, all the cured films of UVCF formulations exhibited excellent adhesive force to the substrate. With the increase of UVCF content, the flexibility gradually decreased, especially for the cured film with $95 \%$ content of UVCF, and the flexibility attained $10 \mathrm{~mm}$, owing to the excess cross-linking structures in the films.

Furthermore, the cross-linking structures also restricted the mobility of the molecular chain, which led to the low impact strength at $31 \mathrm{~kg} \cdot \mathrm{cm}$. As for the cured film with $60 \%$ content of UVCF, the pendulum hardness and impact strength were the highest. This can be explained that, in the cured film, $\mathrm{C}=\mathrm{C}$ double bonds were well polymerized to form the appropriate cross-linking structures with good rubbery and rigid properties. 


\section{Conclusions}

The UV-curable UVCF resin was successfully prepared using IPDI and PETA to modify CF resin. The $M_{n}$ of UVCF resin was about 2010 and the PDI was 2.8. When UV irradiation time was $230 \mathrm{~s}$, the photopolymerization conversion of the UV-curable formulations was above $80 \%$. Due to the crosslinking structure, the cured UVCF film had good thermal stability. Compared with the other cured films, the cured film with $60 \%$ content of UVCF exhibited better overall mechanical properties. The adhesive force, flexibility, pendulum hardness, and impact strength were 1 grade, $4 \mathrm{~mm}, 0.61$ time ratio, and $42 \mathrm{~kg} \cdot \mathrm{cm}$, respectively.

\section{Conflict of Interests}

The authors declare that there is no conflict of interests regarding the publication of this paper.

\section{Acknowledgments}

This work was financially supported by the National Natural Science Foundation of China (Grant no. 51203051), the Fundamental Research Funds for the Central Universities, SCUT (Grant no. 2014ZZ0006), and China Postdoctoral Science Foundation (Grant no. 2013M531842).

\section{References}

[1] P. M. Pihko, K. M. Laurikainen, A. Usano, A. I. Nyberg, and J. A. Kaavi, "Effect of additives on the proline-catalyzed ketonealdehyde aldol reactions," Tetrahedron, vol. 62, no. 2-3, pp. 317328, 2006.

[2] J. Plank and A. Aignesberger, "Acid group-containing hydrophilic co-condensation products of ketone-aldehyde resins," US Patent no. 4,585,853, 1986.

[3] K. Fischer, H. Petersen, H. Kasch, and E. Wistuba, "Preparation of aqueous ketone resin or ketone/aldehyde resin dispersions, and production of surface-coating binders," US Patent no. 4,644,028, 1987.

[4] V. D. Athawale and N. J. Shetty, "Studies on cyclohexanone formaldehyde-styrenated CNSL coatings," Surface Coatings International Part B: Coatings International, vol. 83, no. 4, pp. 168-172, 2000.

[5] H. S. Patel and A. M. Naji, "Studies on methylolated cyclohexanone-formaldehyde resin-epoxy resin condensation products," Polymer: Plastics Technology and Engineering, vol. 49, no. 11, pp. 1121-1127, 2010.

[6] P. Gloeckner, W. Adrejewski, P. H. Bergmann et al., "Ketonealdehyde resins having low water content, high thermal stability and yellowing resistance," US Patent no. 7,101,958, 2006.

[7] A. R. N. Azimi, R. Yahya, and S.-N. Gan, "Improving coating characteristics of palm stearin alkyd by modification with ketone resin," Progress in Organic Coatings, vol. 76, no. 4, pp. 712-719, 2013.

[8] P. Glöeckner, A. Wenning, and P. Denkinger, "Polymer compositions of carbonyl-hydrated ketone-aldehyde resins and polyisocyanates in reactive solvents," US Patent no. 7,700,664, 2010.
[9] H. C. Maytum, B. Tavassoli, and J. M. J. Williams, "Reduction of aldehydes and ketones by transfer hydrogenation with 1,4butanediol," Organic Letters, vol. 9, no. 21, pp. 4387-4389, 2007.

[10] D. Font, A. Bastero, S. Sayalero, C. Jimeno, and M. A. Pericàs, "Highly enantioselective $\alpha$-aminoxylation of aldehydes and ketones with a polymer-supported organocatalyst," Organic Letters, vol. 9, no. 10, pp. 1943-1946, 2007.

[11] Y.-F. Zhang, X.-R. Zeng, and B.-Y. Ren, "Synthesis and structural characterization of urea-isobutyraldehyde- formaldehyde resins," Journal of Coatings Technology Research, vol. 6, no. 3, pp. 337-344, 2009.

[12] D. Glowacz-Czerwonka and M. Kucharski, "New melamineformaldehyde-ketone polymers. V. coatings from melamine and MEK-based reactive solvents," Journal of Applied Polymer Science, vol. 109, no. 4, pp. 2156-2168, 2008.

[13] H. Farzad, F. Najafi, M. Bengisu, E. Yilmaz, and B. S. Hadavand, "Synthesis and characterization of aliphatic tri-functional oligomeric urethane methacrylate used for UV-curable aluminum pigmented coatings," Journal of Macromolecular Science, Part A: Pure and Applied Chemistry, vol. 50, no. 5, pp. 504512, 2013.

[14] F. Bauer, U. Decker, S. Naumov, and C. Riedel, "Photoinitiatorfree UV curing and matting of acrylate-based nanocomposite coatings: part 3," Progress in Organic Coatings, vol. 77, no. 6, pp. 1085-1094, 2014.

[15] C. Bai, X. Zhang, and J. Dai, "Synthesis and characterization of a new UV cross-linkable waterborne siloxane-polyurethane dispersion," Journal of Macromolecular Science, Part A: Pure and Applied Chemistry, vol. 44, no. 11, pp. 1203-1208, 2007.

[16] D. Glöeckner, D. Mindach, and D. Denkinger, "Radiation curable resins based on hydrogenated ketone and/or phenolaldehyde resins and process for their preparation," European Patent no. 1,508,582, 2008.

[17] R. S. Mishra, A. K. Mishra, and K. Raju, "Synthesis and property study of UV-curable hyperbranched polyurethane acrylate/ ZnO hybrid coatings," European Polymer Journal, vol. 45, no. 3, pp. 960-966, 2009.

[18] Q. Gao, H. Li, and X. Zeng, "Preparation and characterization of UV-curable hyperbranched polyurethane acrylate," Journal of Coatings Technology Research, vol. 8, no. 1, pp. 61-66, 2011.

[19] F. Bao and W. Shi, "Synthesis and properties of hyperbranched polyurethane acrylate used for UV curing coatings," Progress in Organic Coatings, vol. 68, no. 4, pp. 334-339, 2010.

[20] H. Li, Y. Zhang, and X. Zeng, "Two-step synthesis and characterization of urea-isobutyraldehyde-formaldehyde resins," Progress in Organic Coatings, vol. 66, no. 2, pp. 167-172, 2009.

[21] Y. Yuan, R. Liu, C. L. Wang, J. Luo, and X. Y. Liu, "Synthesis of UV-curable acrylate polymer containing sulfonic groups for anti-fog coatings," Progress in Organic Coatings, vol. 77, no. 4, pp. 785-789, 2014.

[22] C. Y. Bai, X. Y. Zhang, J. B. Dai, and W. H. Li, "A new UV curable waterborne polyurethane: effect of $\mathrm{C}=\mathrm{C}$ content on the film properties," Progress in Organic Coatings, vol. 55, no. 3, pp. 291-295, 2006.

[23] S. Tasic, B. Bozic, and B. Dunjic, "Synthesis of new hyperbranched urethane-acrylates and their evaluation in UVcurable coatings," Progress in Organic Coatings, vol. 51, no. 4, pp. 320-327, 2004.

[24] Z. Susteric and I. Dimitrievski, "Morphology and finite strain rheology of NBR/TPU blends," International Journal of Polymeric Materials and Polymeric Biomaterials, vol. 52, no. 6, pp. 527-548, 2003. 
[25] J. Shi, X. Yang, Q. Han, X. Wang, and L. Lu, "Synthesis and characterization of polyurethane-coated palygorskite," Applied Clay Science, vol. 46, no. 3, pp. 333-335, 2009.

[26] W. H. Yin, X. R. Zeng, H. Q. Li, Y. J. Hou, and Q. Z. Gao, "Synthesis, photopolymerization kinetics, and thermal properties of UV-curable waterborne hyperbranched polyurethane acrylate dispersions," Journal of Coatings Technology Research, vol. 8, no. 5, pp. 577-584, 2011.

[27] N. Yoshitake and M. Furukawa, “Thermal degradation mechanism of $\alpha, \gamma$-diphenyl alkyl allophanate as a model polyurethane by pyrolysis-high-resolution gas chromatography/FT-IR," Journal of Analytical and Applied Pyrolysis, vol. 33, pp. 269-281, 1995. 

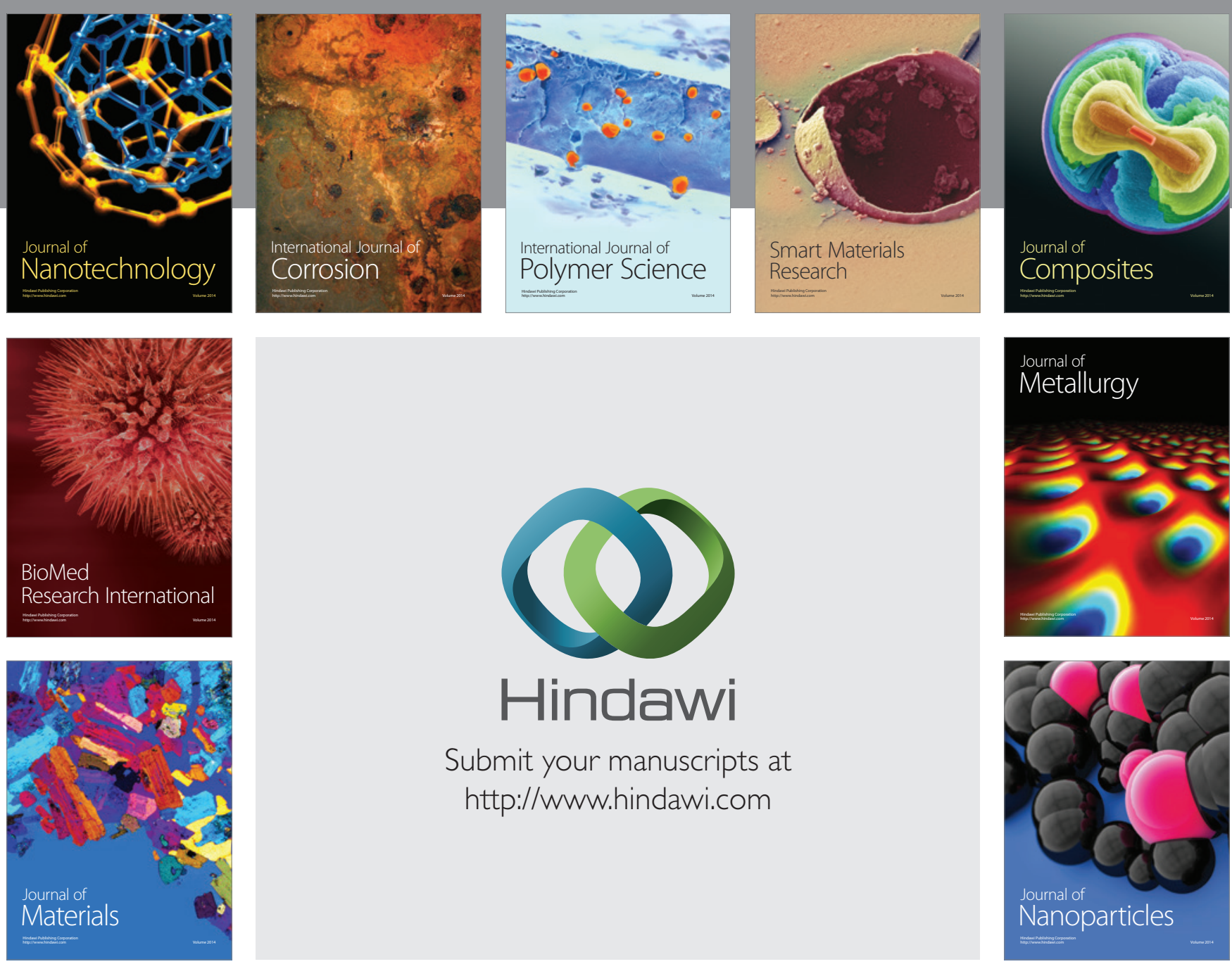

Submit your manuscripts at http://www.hindawi.com
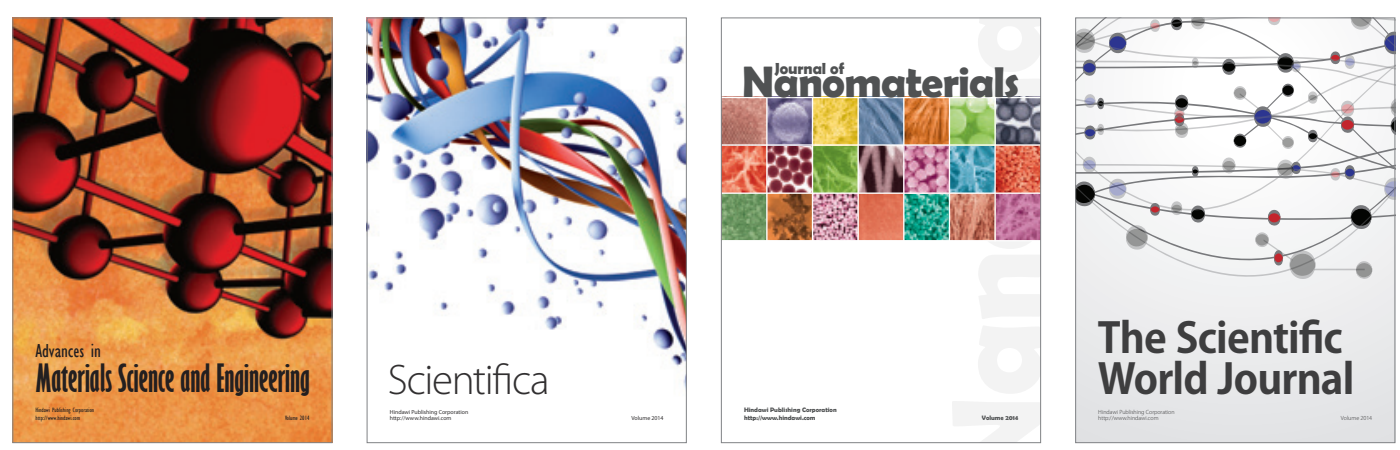

\section{The Scientific World Journal}
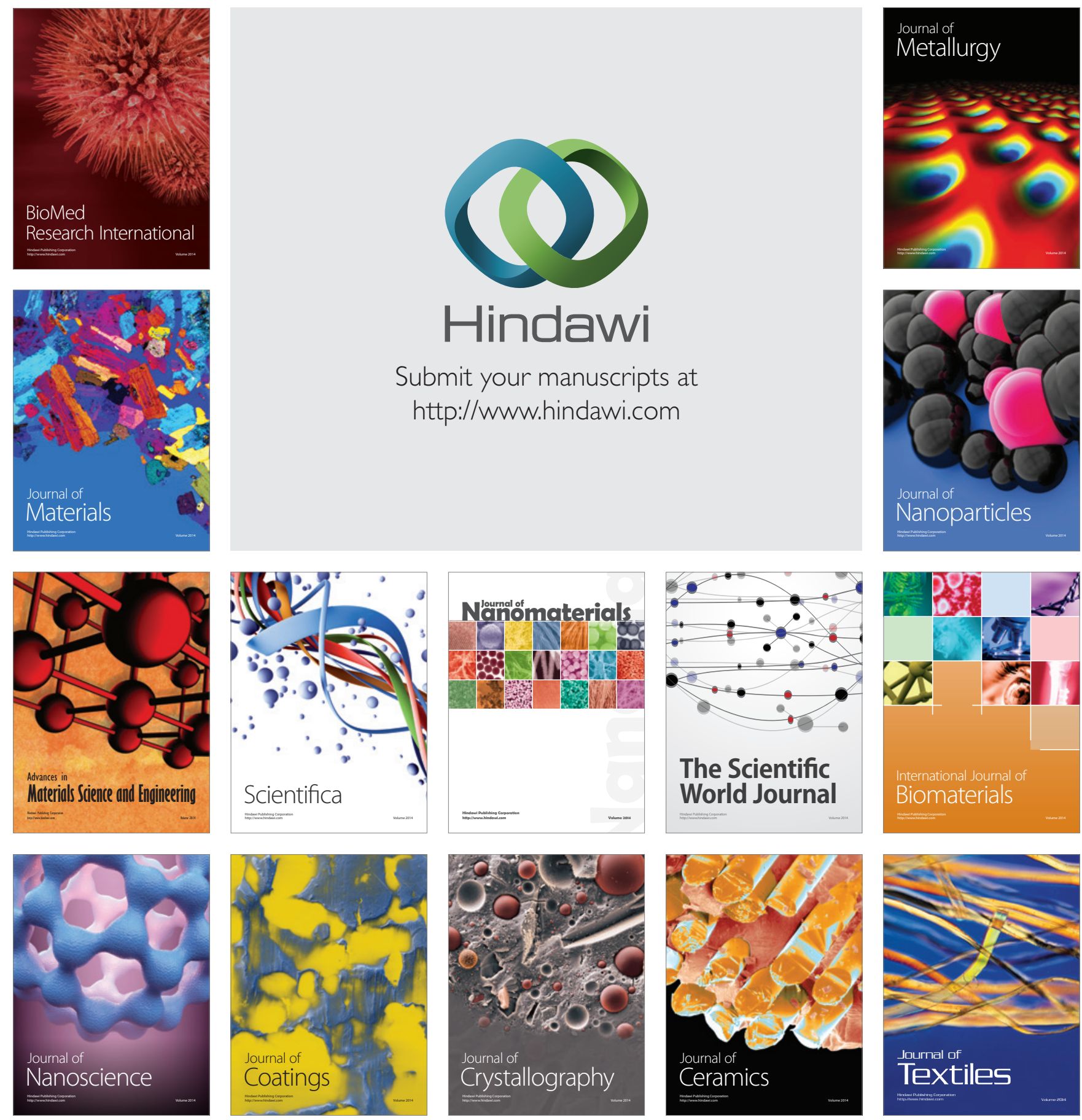